\title{
Buckling load optimization for 2D continuum models, with alternative formulation for buckling load estimation
}

\author{
Pedersen, Niels L.; Pedersen, Pauli
}

Published in:

Structural and Multidisciplinary Optimization

Link to article, DOI:

$10.1007 / \mathrm{s} 00158-018-2030-3$

Publication date:

2018

Document Version

Peer reviewed version

Link back to DTU Orbit

Citation (APA):

Pedersen, N. L., \& Pedersen, P. (2018). Buckling load optimization for 2D continuum models, with alternative formulation for buckling load estimation. Structural and Multidisciplinary Optimization, 58, 2163-2172. https://doi.org/10.1007/s00158-018-2030-3

\section{General rights}

Copyright and moral rights for the publications made accessible in the public portal are retained by the authors and/or other copyright owners and it is a condition of accessing publications that users recognise and abide by the legal requirements associated with these rights.

- Users may download and print one copy of any publication from the public portal for the purpose of private study or research.

- You may not further distribute the material or use it for any profit-making activity or commercial gain

- You may freely distribute the URL identifying the publication in the public portal 


\title{
Buckling load optimization for 2D continuum models, with alternative formulation for buckling load estimation
}

\author{
Niels L. Pedersen • Pauli Pedersen
}

Received: date / Accepted: date

\begin{abstract}
Buckling load estimation of continua modeled by finite element (FE) should be based on nonlinear equilibrium. When such equilibrium is obtained by incremental solutions and when sensitivity analysis as well as iterative redesigns are included, the computational demands are large especially due to optimization. Therefore, examples presented in the literature relate to few design variables and/or few degrees of freedom.

In the present paper a non-incremental analysis is suggested, and a simple sensitivity analysis as well as recursive redesign is proposed. The implicit geometrical non-linear analysis, based on Green-Lagrange strains, apply the secant stiffness matrix as well as the tangent stiffness matrix, both determined for the equilibrium corresponding to a given reference load, obtained by the Newton-Raphson method. For the formulated eigenvalue problem, which solution gives the estimated buckling load, the tangent stiffness matrix is of major importance. In contrast to formulations based on incremental solutions, the tangent stiffness matrix is here divided into two matrices, the stress stiffness matrix that is linear depending on stresses and the remaining part of the tangent stiffness matrix. Examples verify the effectiveness of the proposed procedure.
\end{abstract}

Niels L. Pedersen

Dept. of Mechanical Engineering, Solid Mechanics

Technical University of Denmark

Nils Koppels Allé, Building 404, DK-2800 Kgs. Lyngby, Denmark

E-mail: nlp@mek.dtu.dk

Pauli. Pedersen

Dept. of Mechanical Engineering, Solid Mechanics

Technical University of Denmark

Nils Koppels Allé, Building 404, DK-2800 Kgs. Lyngby, Denmark
Keywords Buckling estimation - Eigenvalue formulations · Analytical · Optimization · Sensitivities · FE

\section{Introduction}

Estimate of buckling load is the background for redesign towards improved buckling load of a finite element (FE) model of a loaded continuum. To the non-linear analysis problem is added iterative redesign, resulting in a challenging problem which contains several iterations: iteration for non-linear equilibrium of a static problem, iteration for solution of an eigenvalue problem and iteration for improved redesign.

The present paper is a follow up of Pedersen and Pedersen (2018) on 3D continuum design with focus on sensitivity analysis. Here the focus is on alternative formulations of the involved eigenvalue problem, on importance of magnitude of reference load, on dependence of imperfections such as eccentricity and the influence of optimized design to control these dependence's. The included references concentrate on these aspects without repeating the references given in Pedersen and Pedersen (2018).

Large computational demands may be reduced by simplicity in formulation. For the static equilibrium this is obtained by assuming linear elastic material, such that implicit convergence using Green-Lagrange strains in a total Lagrangian formulation of the static problem is effective. The application of analytical expressions for secant stiffness matrices as well as for tangent stiffness matrices makes detailed design and analysis modeling possible. The applied analytical sensitivity analysis makes an optimality criterion possible. Up to 100000 elements and design variables for a problem has been 
treated, with possibility for design interaction to obtain a better understanding of the actual analysis and design problem.

Section 2 presents and discuss three alternative formulations for the eigenvalue problem that by solution estimate the buckling load and buckling mode. Thus an optimal redesign is based on an estimate and not on the completely determined instability load. Section 3 includes two examples of uniform (non-optimized) designs for which estimated buckling loads as a function of reference load are determined. Section 4 presents the applied geometrical non-linear analysis based on NewtonRaphson iteration using the secant stiffness matrices, i.e., implicit iterations to full convergence. Section 5 describes the procedures for optimized redesign, i.e., sensitivity analysis, optimality criterion and recursive redesign. Examples of optimization histories and optimized designs follow in Section 6.

\section{Formulations for buckling load estimation}

In order to clarify the suggested formulation in Section 2.3 for buckling load estimation, two alternative formulations are here shortly described. This involves a clarification of the involved stiffness matrices.

\subsection{The simple linear buckling analysis}

In linear buckling estimation as described in Cook et al (2002), the eigenvalue problem that constitutes the background for estimating critical buckling load, is

$$
\left.\left(\left[S_{0}\right]+\lambda\left[S_{\sigma}\right]\right)\right)\{\Delta\}=\{0\}
$$

where $\left[S_{0}\right]$ is the initial global stiffness matrix and $\left[S_{\sigma}\right]$ is the stress stiffness matrix that is part of the tangent stiffness matrix, both obtained based on linear elasticity. The buckling mode $\{\Delta\}$ is corresponding to the eigenvalue $\lambda$. The matrix $\left[S_{\sigma}\right]$ is obtained by a reference load vector $\{\bar{A}\}$ and a factor on $\{\bar{A}\}$ implies the same factor on $\left[S_{\sigma}\right]$. The estimated critical buckling load vector $\{A\}_{C}$ is

$$
\{A\}_{C}=\lambda_{1}\{\bar{A}\}
$$

where $\lambda_{1}$ is the lowest eigenvalue for the eigenvalue problem (1). Comments to this formulation are:

- From the assumption of linearity between $\{\bar{A}\}$ and $\left[S_{\sigma}\right]$ follows directly, that the critical buckling load vector $\{A\}_{C}$ is independent of the norm of $\{\bar{A}\}$. However, in most cases this is not the reality.
- The relations between the individual stress components in an element of a finite element model are unchanged for simple linear buckling analysis. However, with geometrical non-linear displacement analysis this is not the case, even assuming material linear elasticity.

- What is the physical reality behind a linear combination of an initial global stiffness matrix and part of a tangent stiffness matrix? With no good answer to this, the sensitivity analysis based on (1) is questionable from a physical point of view.

From these comments it is decided to concentrate on estimates based on non-linear equilibrium for a given load vector $\{\bar{A}\}$.

2.2 Incremental non-linear equilibrium before buckling estimate

In Brendel and Ramm (1982) and in Bathe and Dvorkin (1983) incremental non-linear solution of a FE continuum is described in details and based on the obtained equilibrium for geometrical as well as material nonlinearity. A linear eigenvalue problem is formulated

$$
\left.\left(\left[S_{t}\right]+\lambda\left[\Delta S_{t}\right]\right)\right)\{\Delta\}=\{0\}
$$

where $\left[S_{t}\right]$ is the final tangent stiffness matrix, $\left[\Delta S_{t}\right]$ is the recent incremental tangent stiffness matrix and $\lambda,\{\Delta\}$ is an eigenvalue pair as in Section 2.1. Comments to this formulation are:

- The recent increment in the tangent stiffness matrix $\left[\Delta S_{t}\right]$ also include the part not linear dependent on the stresses.

- Computational demands with incremental solutions are large, and will further increase when sensitivity analysis and optimal design are included.

- Examples with larger FE models of continua and benchmark tests have not been found in the literature by the authors.

2.3 Buckling estimate by separation of the tangent stiffness matrix

For buckling estimate the approach applied in the present paper is based on the full tangent stiffness matrix $\left[\bar{S}_{t}\right]$ that is separated in a gamma stiffness matrix $\left[\bar{S}_{\gamma}\right]$ and a stress stiffness matrix $\left[\bar{S}_{\sigma}\right]$.

$\left[\bar{S}_{t}\right]=\left[\bar{S}_{\gamma}\right]+\left[\bar{S}_{\sigma}\right]$ 
as described in Section 5.1.2 of Crisfield (1991), using the nomenclature $\left[K_{t}\right]=\left[K_{t 1}\right]+\left[K_{t \sigma}\right]$. The chosen index $\gamma$ here, refer to the dependence on the displacement gradients. With zero gradients and zero stresses $\left[\bar{S}_{\gamma}(\gamma=\sigma=0)\right]=\left[S_{0}\right]=\left[S_{t 0}\right]$. All the element matrices applied in the present paper are based on GreenLagrange strain, they are derived and listed as analytical expressions in Pedersen (2005).

The eigenvalue problem for non-linear buckling analysis is interpreted as an extrapolation along the tangent stiffness matrix

$$
\left(\left[\bar{S}_{\gamma}\right]+\lambda\left[\bar{S}_{\sigma}\right]\right)\{\Delta\}=\{0\}
$$

The matrices depend on a reference load $\{\bar{A}\}$ and therefore the stiffness matrices are with a bar notation and must be determined by iteration, here using the secant stiffness matrix. Comments to the suggested new eigenvalue formulation are:

- As seen from the analytical expressions, the coefficient matrix $\left[\bar{S}_{\sigma}\right]$ to the eigenvalue $\lambda$ depends linear on stresses as evaluated from equilibrium corresponding to the load distribution $\{\bar{A}\}$. Note, that even for the 3D tetrahedron element the matrix $\left[\bar{S}_{\sigma}\right]$ is simple, as listed in Pedersen and Pedersen (2018).

- Only geometrical non-linearity is in the present paper included, and the stiffness matrices are based on Green-Lagrange strains in a total Lagrangian formulation.

- Material non-linearity may be addressed by extending the iterative implicit formulation with load incrementation. This will involve a more extended notation as in Brendel and Ramm (1982) and in Bathe and Dvorkin (1983).

- During Newton-Raphson iterations the residuals are obtained based on the non-symmetric element secant stiffness matrices $\{R\}=\sum_{e}\left(\left[S_{s}\right]_{e}\{D\}_{e}\right)-\{\bar{A}\}$ and an error norm of $10^{-12}$ is often obtained in less than 10 iterations, assuming that the tangent matrix $\left[S_{t}\right]$ is not close to being singular.

Before applying this eigenvalue formulation to optimal design, it is applied to two examples with uniform design. Primarily to show resulting agreement between classical beam buckling and a 2D finite element. For the first example of a cantilever problem, we show also the influence from eccentric load application and more generally the obtained buckling load as a function of reference load. The second example is a $2 \mathrm{D}$ model of the frame problem, known as the Roorda-Koiter frame.

\section{Buckling load as a function of reference load}

The first comment to the simple linear buckling analysis by the eigenvalue problem (1) was that the critical buckling load vector $\{A\}_{C}$ is independent of the norm of the reference load $\{\bar{A}\}$. Therefore results based on the eigenvalue problem (5) are determined for a range of norms for the reference load, to show the influence.

\subsection{A uniform cantilever column with centered and eccentric load}

A 1D cantilever column model of length $L=10 \mathrm{~m}$, cross-sectional height for in plane bending $h=1 \mathrm{~m}$ and uniform thickness $b=0.005 \mathrm{~m}$ (only in plane buckling). Modulus of elasticity $E=2 \cdot 10^{11} \mathrm{~Pa}$ is assumed and the analytical formula for critical column force is

$P_{C}=\frac{\pi^{2}}{4} \frac{E I}{L^{2}} \simeq 2.056 \cdot 10^{6} \mathrm{~N}$

to be compared with a finite element solution for a uniform 2D model containing 16384 triangular elements. The results are presented in Figure 1 for two different cases of a 2D plane model (plane stress and $\nu=0$ ). The agreement with the analytical result is fine for smaller reference loads (the slenderness ratio of the column is about 60$)$.

Buckling load in $10^{6} \mathrm{~N}$

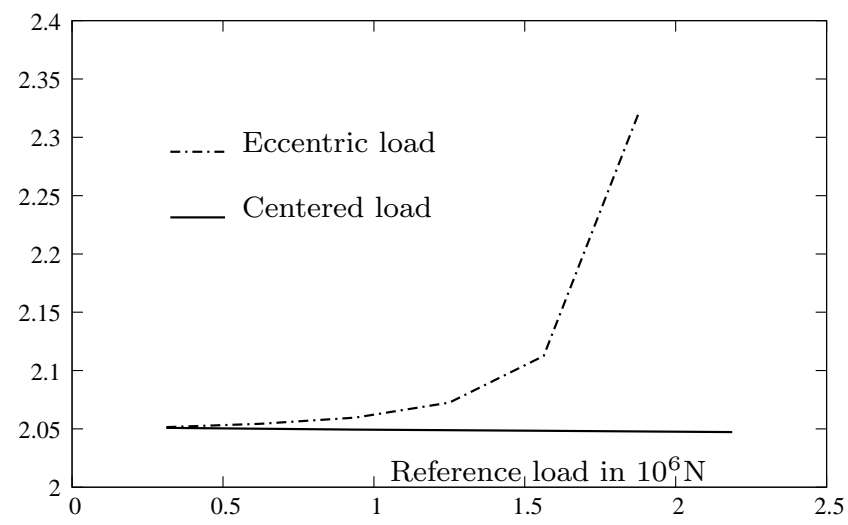

Fig. 1 For a uniform cantilever column with two different load positions, the buckling load as a function of reference load. The geometrical non-linear displacement analysis is based on Green-Lagrange strains.

For centered load the full curve in Figure 1 shows almost constant buckling results and total agreement with the analytical 1D beam results in (6). For this case it was even possible to pass the singularity at 2.05 and obtain an eigenvalue $\lambda_{1}<1$. The results for optimized 

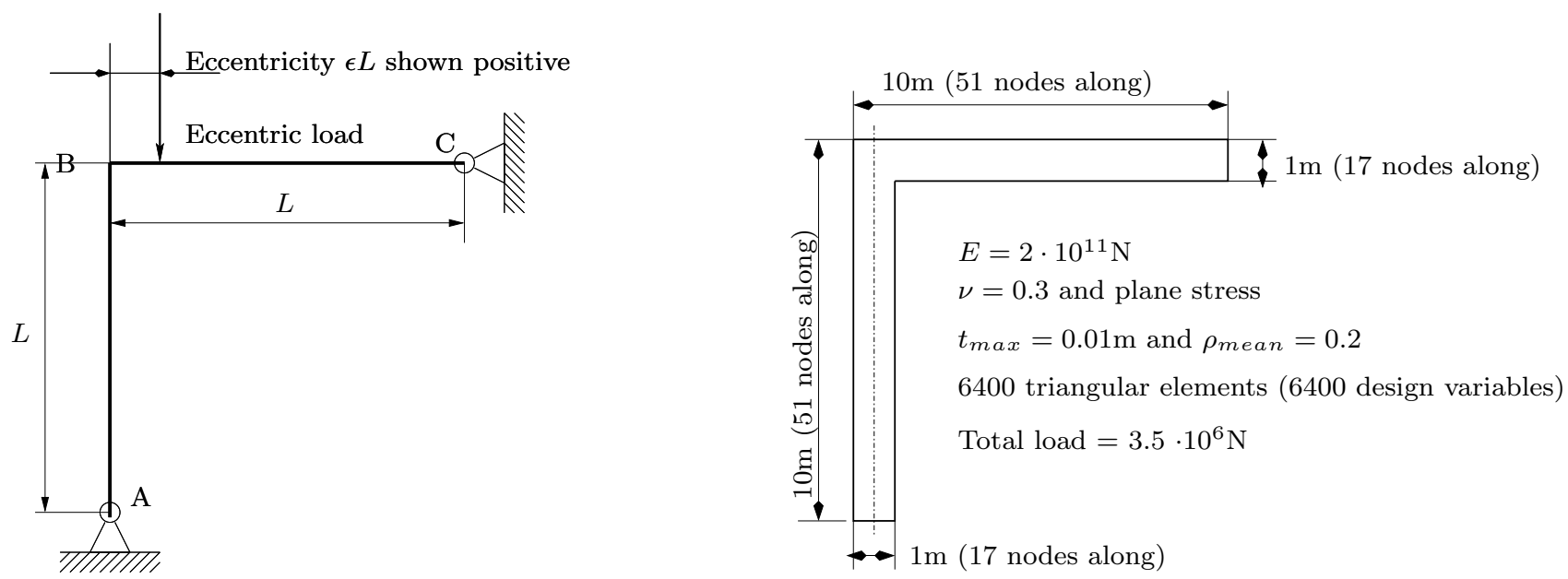

Fig. 2 Simply supported frame model with dimensions at the finite element model to the right. Four different cases of load positions are analyzed, corresponding to $\epsilon=-0.04,-0.02,0,+0.02$.

design of the cantilever column with centered load are presented in Section 6.1.

For eccentric load the hash-dot curve in Figure 1 shows increasing buckling load for increasing reference load. This means that this problem, based on linear elasticity for large displacements, is more like "The Elastica" than a buckling problem. As the formulation of the present paper does not include non-linear material behavior, the problem with eccentric load for this case is not optimized. For the case in Figure 1 with uniform density in the cantilever, an eccentric load will from the load initiation create a bending from the cantilever top in addition to pure compression. Analytical this problem therefore needs more extensive analytical beam-column analysis, than the well known result (6). The results in Figure 1 that are obtained as eigenvalues are confirmed by obtained geometrically non-linear solutions for increased external load cases. The eccentric load case gave elastic equilibrium also for loads higher than (6). The centered column load added a small transverse load at the tip (not to get pure compression solution) results in singular (no convergence) tangent stiffness matrix, close to (6), all in agreement with Figure 1.

\subsection{A uniform frame with different eccentric loads}

A frame model is later subjected to design for maximum buckling load. Figure 2 shows a sketch of the model, known as the Roorda-Koiter frame when modeled with two uniform beam-columns, see Roorda (1965). Here it is for analysis modeled in FE by a number (6400) of 2D plane triangular elements. Note, that the $1 \mathrm{D}$ model is analytically proved unstable for negative imperfection (left eccentric load), but stable for positive imperfection (right eccentric load)

A 2D uniform FE model to be designed in Section 6.2 is here primarily analyzed based on the eigenvalue problem (5) for later discussion of the more general discussion between Figure 3 and the results in Figure 6 . In Figure 3 we note frame buckling for left eccentric load and a stabilized frame for right eccentric load in agreement with the $1 \mathrm{D}$ theoretical result.

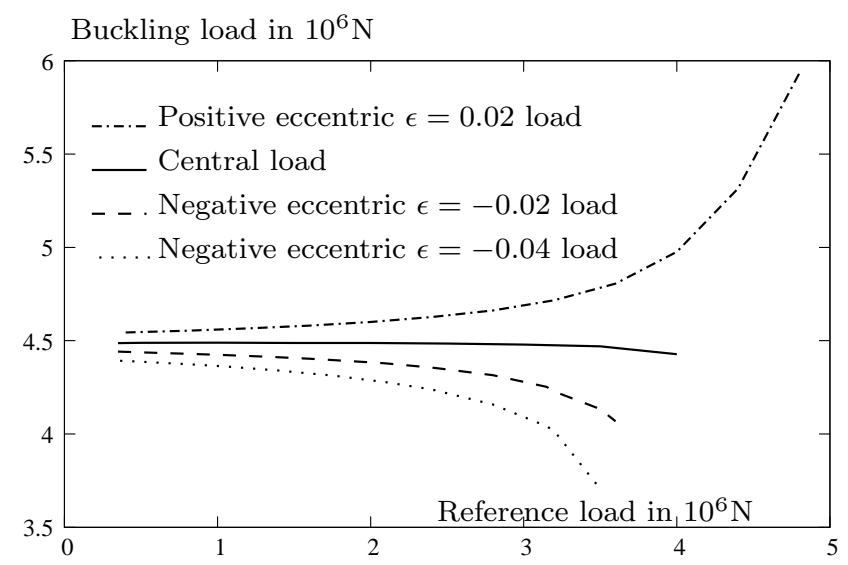

Fig. 3 For a uniform frame with four different load positions, the buckling load as a function of reference loads, based on geometrical non-linear displacement analysis.

For left eccentric load the dotted and the dashed curve in Figure 3 shows decreasing buckling load for increasing reference load until stooped at the singularity of the total tangent stiffness matrix. This behavior is due to a horizontal beam-column in increasing compression, as can be evaluated from the 1D analytical frame. For the centered load the full curve in Figure 3 shows rather constant buckling load until singular- 
ity of the total tangent stiffness matrix. In agreement with the $1 \mathrm{D}$ analytical frame increasing left eccentricity decrease the buckling load.

For right eccentric load the dash-dot curve in Figure 3 shows still increasing buckling load for increasing reference load. This behavior is due to the horizontal beam $\mathrm{BC}$ in increasing tension that stabilizes the frame, as can be evaluated from the 1D analytical frame. This case is not subjected to optimization as only geometrical non-linearity is included in the presented formulation.

\section{Geometrical non-linear analysis}

Before results of optimization are presented, geometrical non-linear analysis, sensitivity analysis and the optimization procedure are described. The first part of necessary analysis for a given design, i.e., after each redesign, is to determine the geometrical non-linear elastic equilibrium as described by

$$
\left[S_{s}\right]\{D\}=\{\bar{A}\}
$$

with

$$
\begin{gathered}
{\left[S_{s}\right]=\sum_{e}\left[S_{s}\right]_{e} \text { where }\left[S_{s}\right]_{e} \text { depends on }\{D\}_{e}} \\
\text { that is part of }\{D\}
\end{gathered}
$$

and where $\{\bar{A}\}$ is a given reference load vector and the non-symmetric secant stiffness matrix $\left[S_{s}\right]$ is a function of the displacement vector $\{D\}$. Therefore, both matrix $\left[S_{s}\right]$ and vector $\{D\}$ depend implicitly on the chosen reference load vector $\{\bar{A}\}$ and are determined by iteration.

Analytical expressions for $\left[S_{s}\right]$ as depending on $\{D\}$ are presented in Pedersen (2005) for the applied 2D triangular element. The use of the secant stiffness matrix $\left[S_{s}\right]$ is seldom commented, even in books on FE, although the matrix non-symmetry is no problems since the resulting system matrix do not need to be accumulated. Discussions on the secant stiffness matrix is presented in Onate (1995) and Onate and Matias (1996). Application of the secant stiffness matrix in sensitivity analysis is recently presented by Wang et al (2015).

For an iterative solution procedure the Newton-Raphson approach is applied and a system residual $\{R\}$ is defined from

$$
\{R\}=\left[S_{s}\right]\{D\}-\{\bar{A}\}=\sum_{e}\left(\left[S_{s}\right]_{e}\{D\}_{e}\right)-\{\bar{A}\}
$$

and then iteratively update the estimate $\{D\}$ by $\{\Delta D\}$ found from

$$
\{R\}+\{\Delta R\}=\{0\} \Rightarrow\{\Delta R\}=-\{R\}=\left[S_{t}\right]\{\Delta D\}
$$

where the tangent stiffness matrix $\left[S_{t}\right]$ also depends on $\{D\}$.

After iterative convergence the equilibrium is satisfied, indicating this by a bar on all involved quantities

$$
\left[\bar{S}_{s}\right]\{\bar{D}\}=\{\bar{A}\}
$$

The solutions $\{\bar{D}\}$ and $\left[\bar{S}_{s}\right]$ are obtained by these NewtonRaphson iterations, where also the symmetric tangent stiffness matrix $\left[\bar{S}_{t}\right]$ is obtained at convergence to (11). The tangent stiffness matrix at non-linear equilibrium is separated in two matrices, both symmetric

$$
\left[\bar{S}_{t}\right]=\left[\bar{S}_{\gamma}\right]+\left[\bar{S}_{\sigma}\right]
$$

\section{Optimality criterion for optimal redesign}

The optimality criterion for optimal redesign is closely related to design sensitivities, which are primarily derived.

\subsection{Sensitivity analysis for load factor}

The eigenvalue problem (5) is solved by subspace iteration. The sensitivity analysis is derived assuming the matrices $\left[\bar{S}_{\gamma}\right]$ and $\left[\bar{S}_{\sigma}\right]$ are constant from the reference load to the estimated buckling load. From obtained monotonous design convergence it is argued that this assumption is meaningful.

Equation (5) is pre multiplied by the transposed buckling eigenmode $\{\Delta\}^{T}$ to give

$U_{\gamma}+\lambda U_{\sigma}=0$ with the defined energies

$U_{\gamma}:=\{\Delta\}^{T}\left[\bar{S}_{\gamma}\right]\{\Delta\}$ and

$U_{\sigma}:=\{\Delta\}^{T}\left[\bar{S}_{\sigma}\right]\{\Delta\}$

and the Rayleigh quotient

$$
\lambda=-\frac{U_{\gamma}}{U_{\sigma}}
$$

Note for the energies in (13), that the stiffness matrices are based on the solution $\{\bar{D}\}$ from (7) while the displacement vector $\{\Delta\}$ is the eigenmode. 
The sensitivity of the buckling eigenvalue with respect to the density $\rho_{e}$ of the local element $e$ is

$\frac{\partial \lambda}{\partial \rho_{e}}=-\frac{\partial \frac{\widehat{U_{\gamma}}}{U_{\sigma}}}{\partial \rho_{e}}-\frac{\partial \frac{U_{\gamma}}{U_{\sigma}}}{\partial \Delta} \frac{\partial \Delta}{\partial \rho_{e}}=-\frac{\partial \frac{\widehat{U_{\gamma}}}{U_{\sigma}}}{\partial \rho_{e}}$

simplified by the stationarity of the Rayleigh quotient with respect to change of the eigenmode and applying a hat notation for gradients with unchanged eigenmode as in Pedersen and Pedersen (2015).

The stationarity of $\lambda$ as a function of buckling mode is not an assumption of independence but only related to the first order partial derivative. In the linear extrapolation from the non-linear static equilibrium, the tangent matrices $\left[\bar{S}_{\gamma}\right]$ and $\left[\bar{S}_{\sigma}\right]$ are fixed and obtained by the current determined displacement vector $\{\bar{D}\}$ from $\left[\bar{S}_{s}\right]\{\bar{D}\}=\{\bar{A}\}$, but $\{\bar{D}\}$ is not explicitly involved in the sensitivity analysis. This is the background for the simple results obtained with the energy eigenvalue formulation where the buckling mode $\{\Delta\}$ and not the displacement vector $\{\bar{D}\}$ is involved. Every new redesign is initiated with a non-linear static analysis, iteratively determining $\left[\bar{S}_{s}\right]$ and $\left[\bar{S}_{t}\right]$. From the examples it is seen that almost monotonous convergence is obtained with about 20 redesign changes. Influence from multiple buckling modes that have equal or nearby eigenvalues is taken care of in later redesigns without extended sensitivity analysis for non-single eigenmodes. Also mode switching is seen in the examples.

Further differentiation of the Rayleigh quotient gives

$\frac{\partial \lambda}{\partial \rho_{e}}=-\frac{\partial \widehat{U}_{\gamma}}{\partial \rho_{e}} \frac{1}{U_{\sigma}}+\frac{\partial \widehat{U}_{\sigma}}{\partial \rho_{e}} \frac{U_{\gamma}}{U_{\sigma}^{2}}=-\frac{1}{U_{\sigma}}\left(\frac{\partial \widehat{U}_{\gamma}}{\partial \rho_{e}}+\lambda \frac{\partial \widehat{U}_{\sigma}}{\partial \rho_{e}}\right)$

where the energies are accumulated from element energies

$U_{\gamma}=\sum_{e}\left(U_{\gamma}\right)_{e}$ and $U_{\sigma}=\sum_{e}\left(U_{\sigma}\right)_{e}$

The stiffness matrices are assumed to depend explicitly only on the local element density $\rho_{e}$ and if this dependence is assumed to be linear proportionality we find

$$
\begin{aligned}
\frac{\partial \lambda}{\partial \rho_{e}} & =-\frac{1}{U_{\sigma}}\left(\frac{\partial\left(\widehat{U}_{\gamma}\right)_{e}}{\partial \rho_{e}}+\lambda \frac{\partial\left(\widehat{U}_{\sigma}\right)_{e}}{\partial \rho_{e}}\right) \\
& =-\frac{1}{U_{\sigma} \rho_{e}}\left(\left(U_{\gamma}\right)_{e}+\lambda\left(U_{\sigma}\right)_{e}\right)=\frac{\left(U_{\sigma}\right)_{e}}{U_{\sigma}} \frac{1}{\rho_{e}}\left(\lambda_{e}-\lambda\right)
\end{aligned}
$$

For other dependence than linear proportionality, see Pedersen and Pedersen (2015) and the application to the 3D buckling design in Pedersen and Pedersen (2018). The simple result (18) is also valid for other finite element models, such as axisymmetric ring elements and 3D tetrahedron elements.

The objective of maximizing the buckling load $\lambda_{1}\{\bar{A}\}$, i.e., maximizing $\lambda_{1}$ subject to a constant unchanged total mass/volume is

Maximize $\lambda_{1}$ for $g=\sum_{e} \rho_{e} V_{e}-V=0$

and the necessary optimization criterion with a constant $\Lambda$ is

$$
\begin{aligned}
\frac{\partial \lambda}{\partial \rho_{e}}= & \Lambda \frac{\partial g}{\partial \rho_{e}}=\Lambda V_{e} \Rightarrow \\
& \frac{\left(U_{\sigma}\right)_{e}}{U_{\sigma}} \frac{1}{\rho_{e} V_{e}}\left(\lambda_{e}-\lambda\right)=\Lambda_{e}=\Lambda
\end{aligned}
$$

where $U_{\sigma}$ may be normalized to 1 by normalizing the buckling mode $\{\Delta\}$. The modification by non-linear interpolation is described in Pedersen and Pedersen (2018).

\subsection{Numerical design procedure for density variables}

Assume that the value of the optimality criterion for element $e$ is termed $\Lambda_{e}$. Then in cases with negative as well as positive ratios $0>\left(\Lambda_{e}\right)_{\min } \leq \Lambda_{e} \leq\left(\Lambda_{e}\right)_{\max }>$ 0 , which is the case for the buckling optimization, the following heuristic procedure has been applied

For positive gradients $\left(\frac{\left.U_{\sigma}\right)_{e}}{U_{\sigma}}\left(\lambda_{e}-\lambda\right)>0\right)$

$$
\left(\rho_{e}\right)_{\text {new }}=\left(\rho_{e}\right)_{\text {current }}\left(1+4.0 \Lambda_{e} / \Lambda_{\max }\right)^{q} \mathrm{~F}
$$

For negative gradients $\left(\frac{\left.U_{\sigma}\right)_{e}}{U_{\sigma}}\left(\lambda_{e}-\lambda\right)<0\right)$

$$
\left(\rho_{e}\right)_{\text {new }}=\left(\rho_{e}\right)_{\text {current }}\left(1-0.8 \Lambda_{e} / \Lambda_{\text {min }}\right)^{q} F
$$

where the values of $\Lambda_{\min }<0, \Lambda_{\max }>0$ are determined during the evaluation of the gradients. Specific used values in (21) are 4.0,0.8, and $q=0.5$ or $q=0.25$, chosen from experience, acting as a kind of move-limits that influence the number of recursive redesigns (number of eigenvalue analysis). For the procedure (21) the size limits of the non-dimensional density variables

$0<\rho_{\min } \leq \rho_{e} \leq \rho_{\max } \leq 1$

are satisfied iteratively in the "inner" iteration loop without further analysis and sensitivity analysis. The 
Buckling loads in $10^{6} \mathrm{~N}$

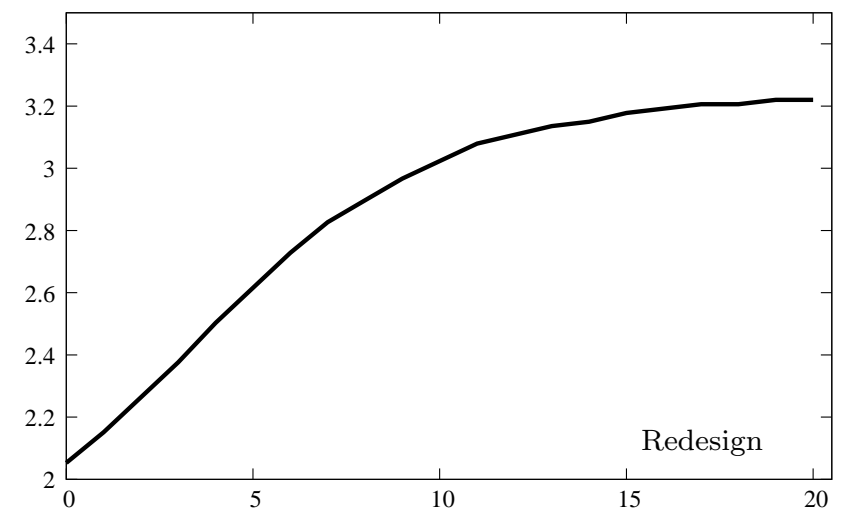

Fig. 4 Results of optimized redesigns with relaxation power $q=0.5$ in the procedure (21) and $50 \%$ material. The model has 16384 elements (16384 design variables). The second buckling load is well above the first one and therefore not shown.

factor $F$ thereby satisfy both the size limits (22) and the specified total amount of material/volume $V$ by $\sum_{e} \rho_{e} V_{e}=V$. In Pedersen and Pedersen (2012) Section 5 on "Redesign towards strength uniformity" describe details for the "inner" iteration, including stop criterion.

The background for the heuristic algorithm (21) is:

- The optimality element criterion (20) is a necessary condition for the elements where size limits (22) are not active. The optimization problem (19) for these elements is then changed to locate a density distribution that imply constant $\Lambda_{e}$ as defined in (20).

- The algorithm (21) always improve density for positive gradients and always decrease density for negative gradients. The influence of the factor $F$ is by a general scaling, always to redesign with unchanged given volume/material. This is obtained by a simple "inner" iteration loop (Pedersen and Pedersen (2012)). During this iteration the active size limits are located and respected. Althought it is not mathematical proven, we have without problems applied the approach (21) for several rather different problems reported in published papers.

- With respect to the chosen factors in (21), the exponential relaxation factor $q$ is chosen to restrict density changes, with $q=0.5$ as a reasonable choice. The other factors 4.0 and 0.8 make it possible to get local density factors for increase from 1 to $5^{q}$ and for decrease from 1 to $0.2^{q}$.

\section{Optimization histories and results for examples}

For the cantilever problem with centered load the density distribution of the FE model is now optimized and the design converges in 20 iterations. For the FE model of a frame with three different load cases are treated; two with initial destabilizing eccentricity and with centered column load. The three optimized density distributions are different and in all three cases rather uniform values of optimality criterion are obtained after 30 improved design for both eccentric and centered load cases. The redesign histories show monotonously increasing buckling load, but convergence first after about 80 iterations, with switching buckling modes.

\subsection{Optimized results for a symmetric loaded cantilever}

The cantilever column case is optimized through 20 redesigns, based on $50 \%$ material $\left(\rho_{\text {mean }}=0.5\right)$ and relaxation power $q=0.5$ in the redesign approach (21). The obtained monotonous convergence is shown in Figure 4 for this case where the second eigenvalue $\lambda_{2}$ is much larger than the first eigenvalue $\lambda_{1}$ (from which the buckling load follows).

Figure 5 shows the resulting design after 20 redesigns together with the distributions of local optimality criterion values, to the left for the initially uniform design and to the right for the optimized design. The resulting design has as expected a highly improved bending stiffness closer to the fixed support with the limit density size $\rho=1$ and less material at the free end and in the middle of the cantilever beam. The initial distribution of optimality criterion values is non uniform, indicating that optimization is possible, and to some extent this distribution reflects the optimized design by showing where to add material and where to remove material. The final distribution of optimality criterion values is almost constant where the density size limits are not reached, indicating that the optimality criterion is fulfilled. The optimality criterion values are positive when the upper limit is reached at $\rho_{e}=1$.

\subsection{Optimized and stabilized results for a frame}

Figure 2 shows the model for the Roorda-Koiter inspired frame, see Roorda (1965). Non-uniform values of optimality criterion indicate that improved load factor before buckling may be obtained by redesign of density distribution. As the difference between optimality criterion and the sensitivity $\frac{\partial \lambda}{\partial \rho_{e}}$ is only the factor $\left(V_{e}\right)^{-3}$, 

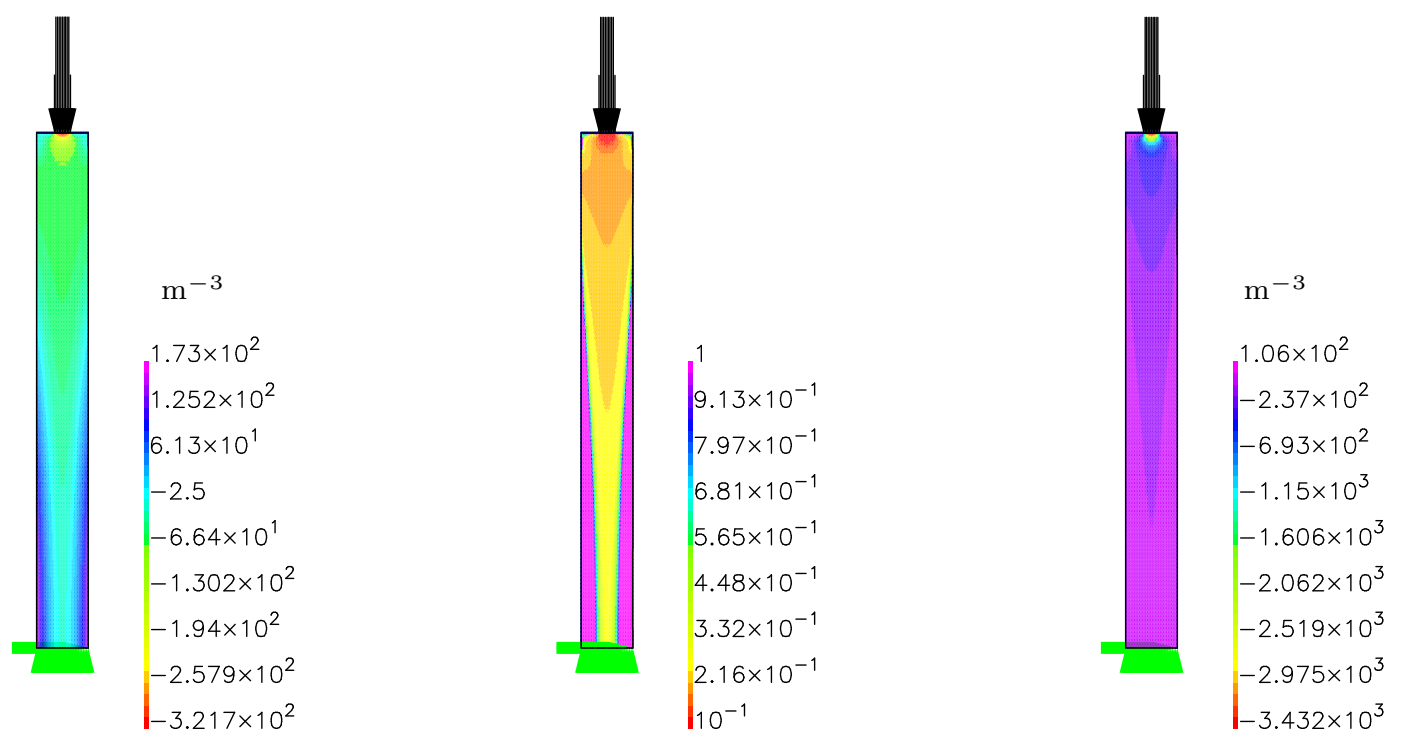

Fig. 5 In the middle the resulting design after 20 redesigns together with the distributions of local optimality criterion values in $\mathrm{m}^{-3}$, to the left for the initially uniform design and to the right for the optimized design. In the design illustration the magenta domains has reached the maximum size of $\rho=1$ and still has positive sensitivities as shown to the right. Minimum size of $\rho=0.1$ are illustrated by red at the top and result in negative sensitivities.

then the distributions shown in Figures 7, 8 and 9 also give a picture of the sensitivities when the element reference volumes are the same.

Buckling loads in $10^{6} \mathrm{~N}$

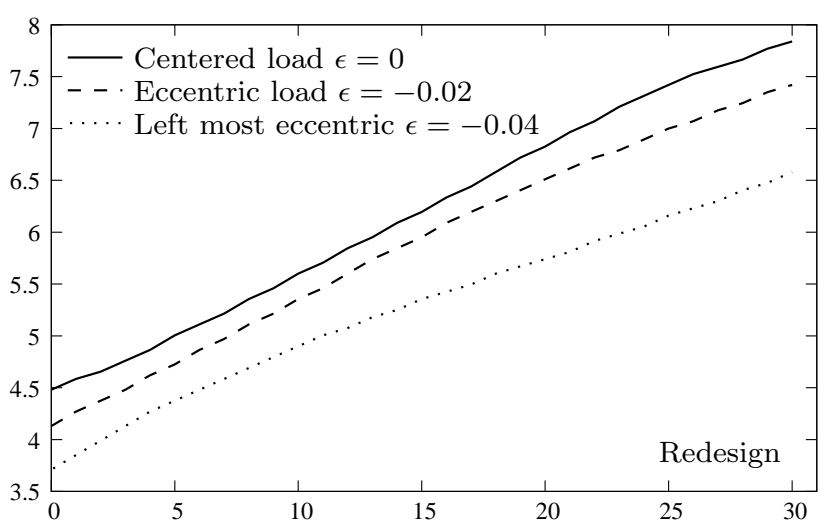

Fig. 6 Design history by eigenvalues evolution with $\lambda_{1}$ being the objective. Three different load positions. Reference load $3.5 \cdot 10^{6} \mathrm{~N}$.

Figure 6 shows monotonously improved buckling load through 30 redesigns for a problem with lower size limit $\left(\rho_{e}\right)_{\min }$ equal to 0.1 , i.e., in reality a kind of optimal stiffening of an initial uniform design. Note, that all three load positions are stabilized, just as for right eccentric load. For each of the three position cases, it is below shown how the redesigns are able to change com- pressive reaction in the horizontal part $\mathrm{BC}$ to tensile stabilizing reaction force. This is shown in Figures 7, 8 and 9 .

Figure 7 shows results for the first case of frame optimization, i.e., the case of left eccentric load at $\epsilon=$ -0.04 . The initial uniform design is shown yellow (top left), corresponding to a uniform design of density $\rho=$ 0.2. External loads are red while reactions at the two simple supports are black. Note compressive reaction at $C$ (in Figure 2), corresponding to norm of the reference load equal to $3.5 \cdot 10^{6} \mathrm{~N}$ which by the dotted curve in Figure 3 gives buckling load close to $3.7 \cdot 10^{6} \mathrm{~N}$. For the uniform design the displaced equilibrium is shown in true scale with the color distribution (top right) presenting the distribution of criterion values and being non-uniform this illustrate the possibility for further increase of buckling load by redesign (here no size limits active). After 30 redesigns following the dotted curve in Figure 6 the resulting density distribution is shown in Figure 7 (bottom left) with corresponding changed distribution of criterion values in Figure 7 (bottom right). The changed reactions after final redesign now include a tensile force from the reaction. The optimized design has therefore in addition to changed stiffness distribution, also changed the influence of eccentricity. The distribution of optimality criterion values after final redesign is approaching uniform, indicating that the optimality criterion is closer to being fulfilled, except for 

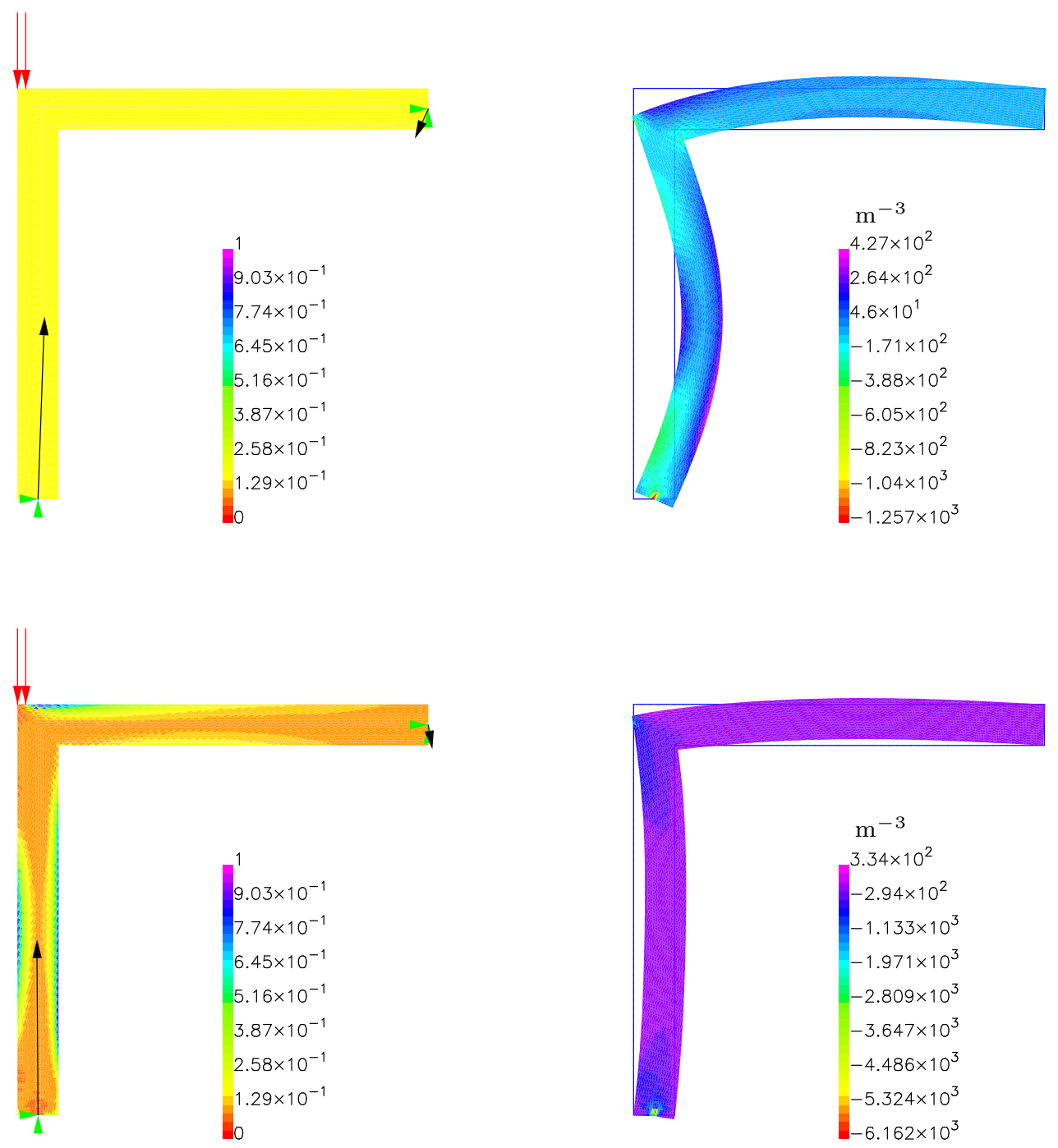

Fig. 7 For the case of larger eccentric load with $\epsilon=-0.04$, the initial uniform design and optimized design after 30 redesigns. Left the density distributions and right the corresponding values of optimality criterion $\left(\Lambda_{e}=\frac{\partial \lambda}{\partial \rho_{e}} \frac{1}{V_{e}}\right)$. Upper row for initial design and lower row for the optimized design. The displacements from the non-linear analysis with load $3.5 \cdot 10^{6} \mathrm{~N}$ are not scaled.

domains with active size constraints $\left(\rho_{e}\right)_{\max }=1$ and $\left(\rho_{e}\right)_{\min }=0.1$.

Figure 8 shows results for the second case of optimized frame design, i.e., the case of less eccentric load $(\epsilon=-0.02)$, here omitting results for the uniform design as in Figure 7. Comparing the optimized results of Figure 8 and of Figure 7 we conclude that there is no big difference.

Figure 9 shows results for the third case of optimized frame design, i.e., the case of centered load $(\epsilon=0)$, here also omitting results for the uniform design as in Figure 7. Comparing the optimized results of Figure 9 and of Figure 7 we note less bending and more symmetric design for the vertical column $A B$.

For the three optimized design, no final convergence as shown in Figure 6 are obtained in 30 redesigns, and for all the cases the redesigns has changed the destabilization by compressive force in the horizontal beamcolumn $B C$ towards stabilization by tensile normal force. For the case of initially lowest buckling load in Figure 7 the number of redesigns are therefore increased above 30 and the design history in Figure 10 is obtained. In this figure also the second order eigenvalue for buckling load is included, and mode switching is noted after 42 redesigns. Convergence to a double buck- 

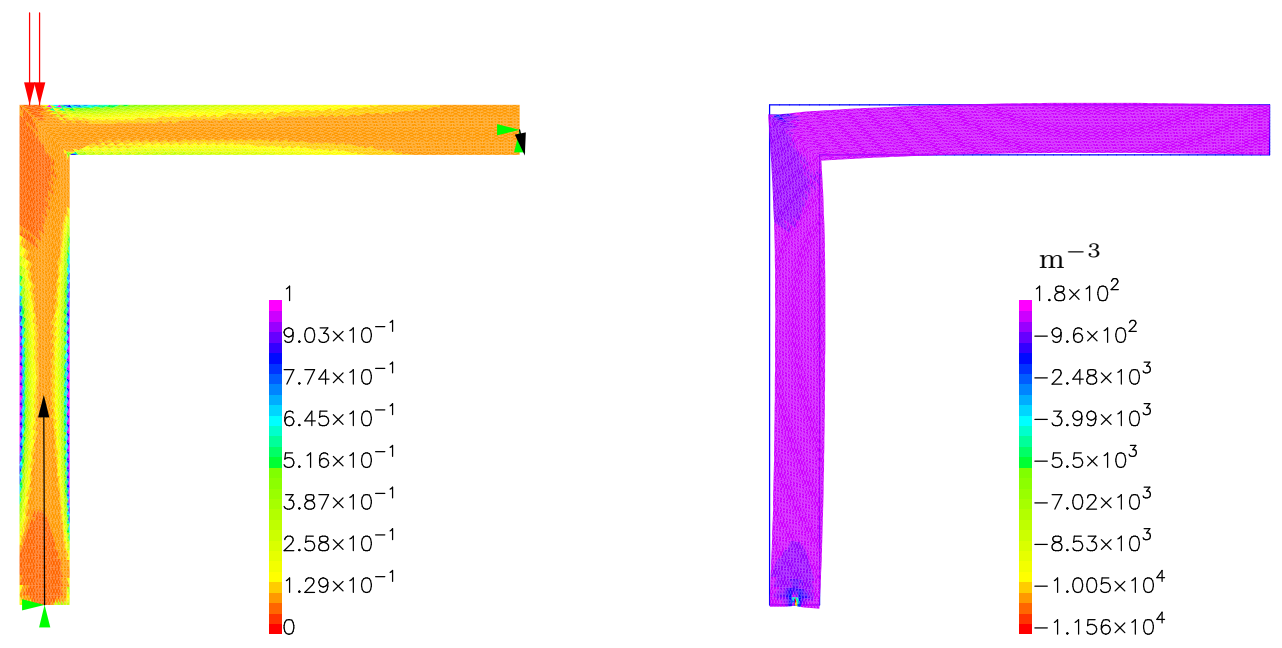

Fig. 8 For the case of less eccentric load $(\epsilon=-0.02)$, the optimized design after 30 redesigns. Left the density distributions and right the corresponding values of optimality criterion $\left(\Lambda_{e}=\frac{\partial \lambda}{\partial \rho_{e}} \frac{1}{V_{e}}\right)$. Resulting displacements from the non-linear analysis from load $3.5 \cdot 10^{6} \mathrm{~N}$ are not scaled.
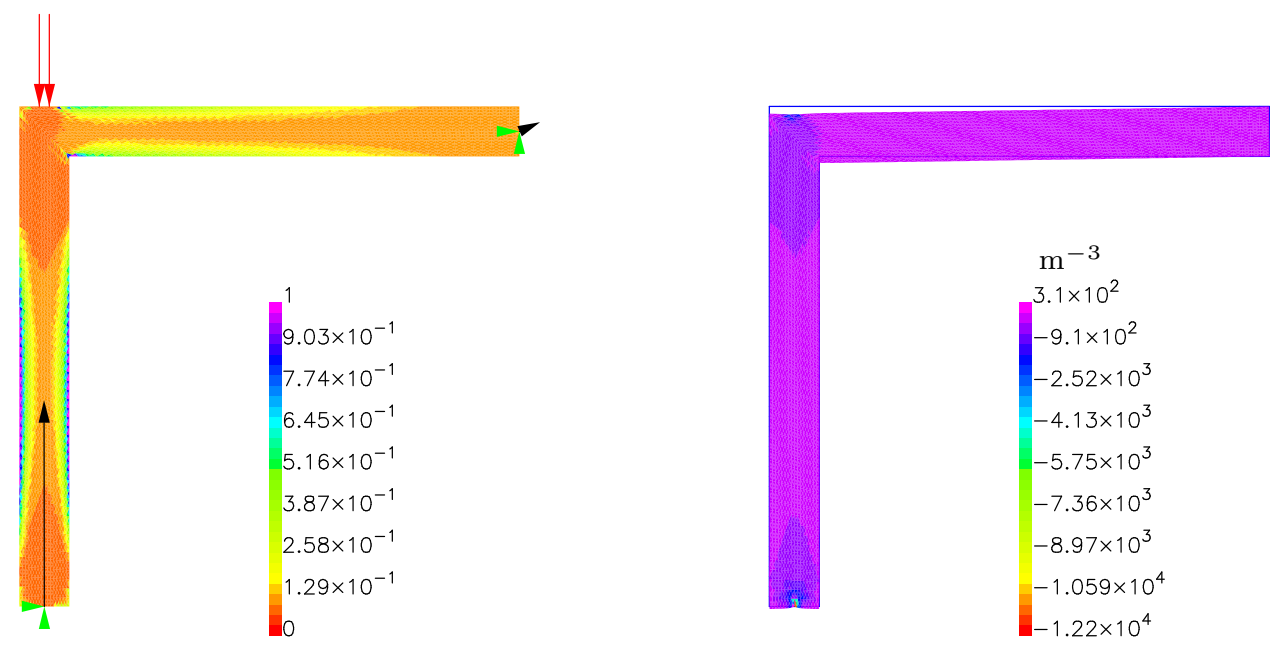

Fig. 9 For the case of centered load, the optimized design after 30 redesigns. Left the density distributions and right the corresponding values of optimality criterion $\left(\Lambda_{e}=\frac{\partial \lambda}{\partial \rho_{e}} \frac{1}{V_{e}}\right)$. Resulting displacements from the non-linear analysis from load $3.5 \cdot 10^{6} \mathrm{~N}$ are not scaled.

ling mode seems to be the result, and the buckling load is more than twice the initial value. It is again noted that the optimization procedure as in Pedersen and Pedersen (2018) is able to improve the design rather monotonously also with mode switching and multiple eigenvalues, without more extended sensitivity analysis.

\section{Conclusions}

Buckling optimization for a continuum finite element model is a challenging problem, for which many aspects are discussed in the present paper.

- Estimation of buckling load and buckling mode may be obtained by an eigenvalue problem. Alternative 
formulations of such eigenvalue problems are discussed, and a suggested version is described and applied for 2D problems.

- A state of equilibrium corresponding to a given reference load needs in general to be determined nonlinearly, and then the involved eigenvalue matrices are determined from the obtained displacement field. The estimated buckling load depends on the magnitude of the reference load, and from a study of this dependence valuable information about the behavior of the non-linear problem are gained. In the present paper only linear material behavior is included and Green-Lagrange strains are used for the geometrical non-linearity.

- For each redesign the non-linear equilibrium are in the present paper obtained by Newton-Raphson iterations without load incrimination's, using current element secant stiffness matrices and current system tangent stiffness matrix. With this approach the computational demands are acceptable. However, adding material non-linearity an incremental approach must be incorporated.

- Simplicity of optimal redesign is obtained by determined analytical sensitivities and from use of an optimality criterion. This is obtained by separating the tangent stiffness matrix into the stress stiffness matrix and the remaining part of the tangent stiffness matrix, here named the displacement gradient $(\gamma)$ matrix.

- The obtained monotonously convergence for different problems shows that linear extrapolation from reference load to buckling load is meaningful, and the sensitivity analysis is robust with respect to switching and multiplicity of buckling modes without the need for a more extended formulation.

- From design for problems with eccentric loads it is found that redesign may change the actual load case from destabilization to stabilization, which is another aspects than the direct stiffness optimization.

- With possible interactive redesign it is possible to update the reference load, although not done in the present examples.

\section{References}

Bathe KJ, Dvorkin EN (1983) On the automatic solution of nonlinear finite element equations. Computers and Structures 17(5-6):871-879

Brendel B, Ramm E (1982) Nichtlineare stabilitatsuntersuchungen mit methode der finiten elemente. Ingenieur-Archiv 51:337-362

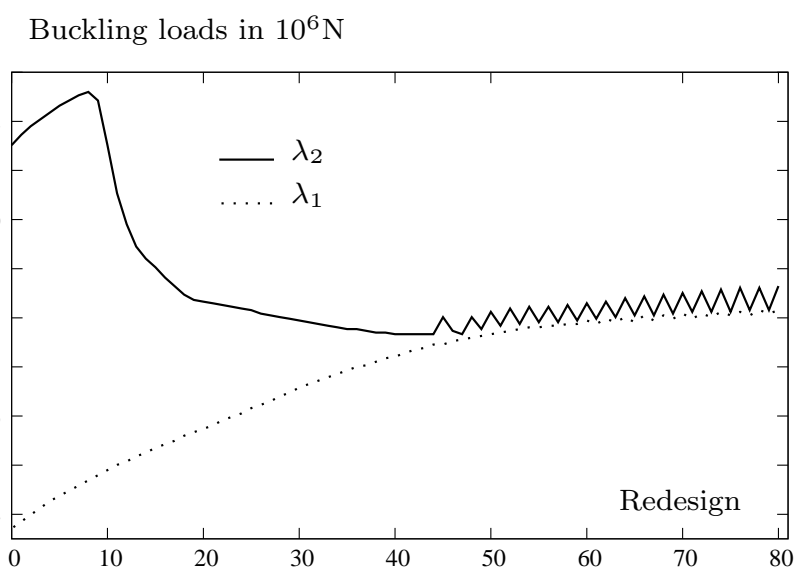

Fig. 10 Design history by eigenvalues evolution with $\lambda_{1}$ being the objective. Switching of buckling mode are seen after 42 redesigns, but it is still possible to increase the lowest buckling load, indicating for the case of $\epsilon=-0.04$ a double buckling mode.

Cook RD, Malkus DS, Plesha ME, Witt RJ (2002) Concepts and Applications of Finite Element Analysis, 4th edn. Wiley, New York, USA, 719 pages

Crisfield MA (1991) Non-linear Finite Element Analysis of Solids and Structures, vol 1. Wiley, Chichester, UK, 345 pages

Onate E (1995) On the derivation and possibilities of the secant stiffness matrix for non linear finite element analysis. Computational Mechanics 15:572-593

Onate E, Matias WT (1996) A critical displacement approach for predicting structural instability. Comput Methods Appl Mech Engrg 134:135-161

Pedersen NL, Pedersen P (2018) Local analytical sensitivity analysis for design of continua with optimized 3D buckling behaviour. Struct Multidisc Optim 57(1):293-304

Pedersen P (2005) Analytical stiffness matrices with Green-Lagrange strain measure. Int J Numer Meth Engng 62:334-352

Pedersen P, Pedersen NL (2012) Interpolation/penalization applied for strength designs of $3 \mathrm{~d}$ thermoelastic structures. Struct Multidisc Optim 45:773-786

Pedersen P, Pedersen NL (2015) Eigenfrequency optimized 3D continua, with possibility for cavities. Journal of Sound and Vibration 341:100-115

Roorda J (1965) Stability of structures with small imperfections. ASCE 91(EM1):87-106

Wang W, Clausen PM, Bletzinger KU (2015) Improved semi-analytical sensitivity analysis using a secant stiffness matrix for geometric nonlinear shape optimization. Computers and Structures 146:143-151 